\title{
See All by Looking at A Few: Sparse Modeling for Finding Representative Objects
}

\author{
Ehsan Elhamifar \\ Johns Hopkins University
}

\author{
Guillermo Sapiro \\ University of Minnesota
}

\author{
René Vidal \\ Johns Hopkins University
}

\begin{abstract}
We consider the problem of finding a few representatives for a dataset, i.e., a subset of data points that efficiently describes the entire dataset. We assume that each data point can be expressed as a linear combination of the representatives and formulate the problem of finding the representatives as a sparse multiple measurement vector problem. In our formulation, both the dictionary and the measurements are given by the data matrix, and the unknown sparse codes select the representatives via convex optimization. In general, we do not assume that the data are lowrank or distributed around cluster centers. When the data do come from a collection of low-rank models, we show that our method automatically selects a few representatives from each low-rank model. We also analyze the geometry of the representatives and discuss their relationship to the vertices of the convex hull of the data. We show that our framework can be extended to detect and reject outliers in datasets, and to efficiently deal with new observations and large datasets. The proposed framework and theoretical foundations are illustrated with examples in video summarization and image classification using representatives.
\end{abstract}

\section{Introduction}

In many areas of machine learning, computer vision, signal/image processing, and information retrieval, one needs to deal with massive collections of data, such as databases of images, videos, and text documents. This has motivated a lot of work in the area of dimensionality reduction, whose goal is to find compact representations of the data that can save memory and computational time and also improve the performance of algorithms that deal with the data. Moreover, dimensionality reduction can also improve our understanding and interpretation of the data.

Because datasets consist of high-dimensional data, most dimensionality reduction methods aim at reducing the feature-space dimension for all the data, e.g., PCA [25], LLE [34], Isomap [36], Diffusion Maps [7], etc. However, another important problem related to large datasets is to find a subset of the data that appropriately represents the whole dataset, thereby reducing the object-space dimension. This is of particular importance in summarizing and visualizing large datasets of natural scenes, objects, faces, hyperspectral data, videos, and text. In addition, this summarization helps to remove outliers from the data as they are not true representatives of the datasets. Finally, memory requirement and computational time of classification and clustering algorithms improve by working on a reduced number of representative data as opposed to a large number of data.

Prior Work. To reduce the dimension of the data in the object-space and find representative points, several methods have been proposed [19, 21, 26, 27, 38]. However, most algorithms assume that the data are either distributed around centers or lie in a low-dimensional space. Kmedoids [26], which can be considered as a variant of Kmeans, assumes that the data are distributed around several cluster centers, called medoids, which are selected from the data. Kmedoids, similar to Kmeans, is an iterative algorithm that strongly depends on the initialization. When similarities/dissimilarities between pairs of data are given and there is a natural clustering based on these similarities, Affinity Propagation [19], similar to Kmedoids, tries to find a data center for each cluster using a message passing algorithm. When the collection of data points is low-rank, Rank Revealing QR (RRQR) algorithm [5, 6] tries to select a few data points by finding a permutation of the data that gives the best conditioned submatrix. The algorithm has suboptimal properties, as it is not guaranteed to find the globally optimal solution in polynomial time, and also relies on the low-rankness assumption. In addition, randomized algorithms for selecting a few columns from a low-rank matrix have been proposed [38]. For a low-rank matrix with missing entries, [2] proposes a greedy algorithm to select a subset of the columns. For a data matrix with nonnegative entries, [17] proposes a nonnegative matrix factorization using an $\ell_{1} / \ell_{\infty}$ optimization to select some of the columns of the data matrix for one of the factors.

Paper Contributions. In this work, we study the problem of finding data representatives using dimensionality reduction in the object-space. We assume that there is a subset 


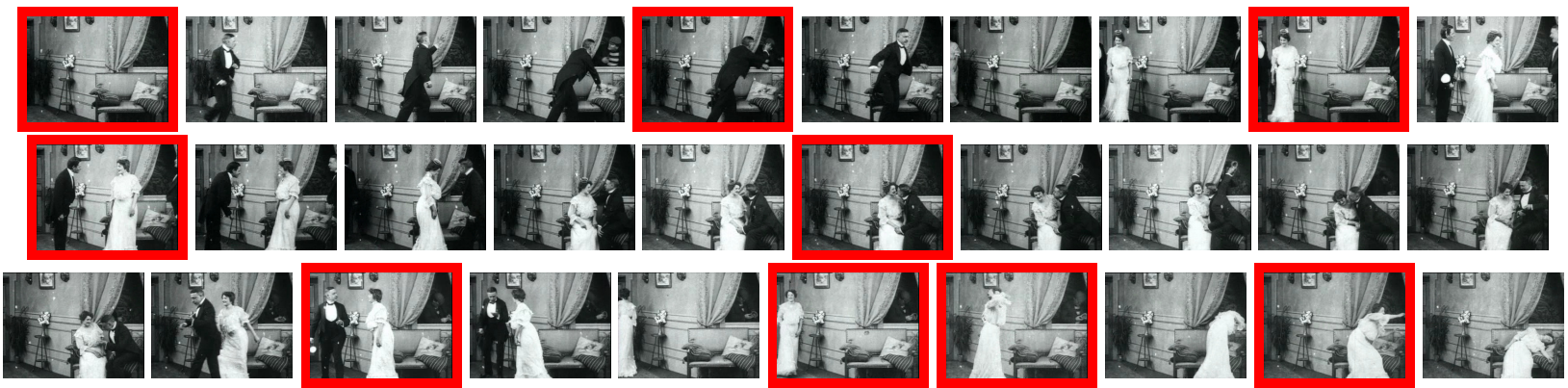

Figure 1. Some frames of the Society Raffles video and the automatically computed representatives of the whole video sequence using our algorithm. The representatives summarize the video as follows: 1) there is a nicely-decorated living room, with a door stage left and a settee in front of an open window in the foreground; 2) a man in the room is talking to someone across the window; 3) a couple enter the room, a man and a woman who is wearing a white gown, and a jeweled tiara. Someone, probably the first man, is standing on the other side of the room; 4) the man who entered with the woman is talking to her and bowing, probably he wants to leave; 5) the first man is sitting with the woman and is reaching for her tiara; 6) the first man is leaving the room, a person is standing across the window and examining the tiara; 7) the woman is entering back to the living room, so she had followed the first man to the door; 8) the woman is clutching her head seeing the bandit across the window; 9) the woman is fainting on the sofa and the bandit has disappeared.
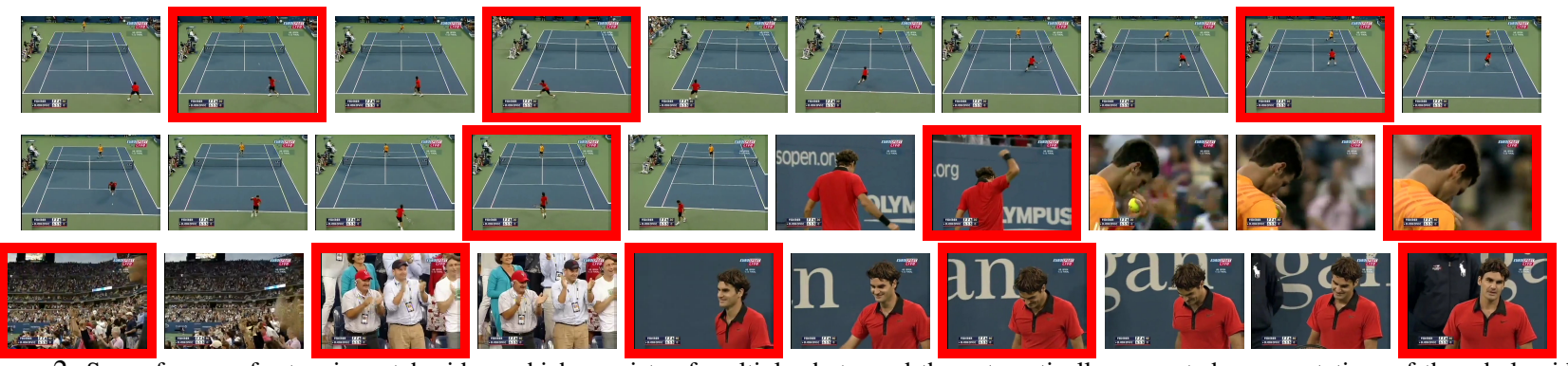

Figure 2. Some frames of a tennis match video, which consists of multiple shots, and the automatically computed representatives of the whole video sequence using our algorithm. Depending on the amount of activities in each shot of the video, we obtained one or a few representatives for that shot.

of data points, called representatives, such that each point in the dataset can be described as a linear combination of a few of the representative points. More specifically, collecting $N$ data points of a dataset in $\mathbb{R}^{m}$ as columns of a data matrix $Y \in \mathbb{R}^{m \times N}$, we consider the optimization problem

$$
\min \|\boldsymbol{Y}-\boldsymbol{Y} \boldsymbol{C}\|_{F}^{2} \quad \text { s.t. }\|\boldsymbol{C}\|_{\text {row }, 0} \leq k, \mathbf{1}^{\top} \boldsymbol{C}=\mathbf{1}^{\top},
$$

where $C \in \mathbb{R}^{N \times N}$ is the coefficient matrix and $\|C\|_{\text {row }, 0}$ counts the number of nonzero rows of $\boldsymbol{C}$ [24, 37]. In other words, we wish to find at most $k \ll N$ representatives that best reconstruct the data collection. This can be viewed as a sparse dictionary learning scheme $[1,30,33]$ where the atoms of the dictionary are chosen from the data points and, instead of letting the support for the sparse codes be arbitrary, we enforce them to have a common support.

The self-expressiveness property, $\boldsymbol{Y}=\boldsymbol{Y} \boldsymbol{C}$, has been studied for subspace clustering using sparse representation [11, 15] and low-rank representation [18, 29]. However, these algorithms are not targeted at finding representatives because of the norms they use for $C$. A framework similar to that in (1), with a nonnegativity constraint on $C$ and without the affine constraint, has been used for nonnegative matrix factorization for the problem of hyperspectral imaging endmember identification [17], without the analysis of the selected columns. In the context of dictionary learning,
[4] and [31] use $\|\boldsymbol{C}\|_{\text {row }, 0}$ to design compact dictionaries and to select similar patches in an image, respectively.

In this work, we propose an algorithm for solving a convex relaxation of (1) and provide an analysis of the theoretical guarantees of the algorithm. Our work has the following contributions with respect to the state of the art:

- Unlike prior works, we do not assume that the data are low-rank or distributed around cluster centers. We only require the total number of representatives to be much smaller than the number of actual points in the dataset.

- When the data come from a collection of low-rank models, we show that our method automatically selects a few data points from each model.

- We analyze the geometry of representatives and show that they correspond to vertices of the convex hull of the data.

- We propose a framework to detect and reject outliers from the dataset using the solution of the proposed optimization program. We also show how to deal with new observations and large datasets efficiently.

- We demonstrate the proposed framework in applications to video summarization (Figs. 1-2) and classification using representatives. 


\section{Problem Formulation}

Consider a set of points in $\mathbb{R}^{m}$ arranged as the columns of the data matrix $\boldsymbol{Y}=\left[\begin{array}{lll}\boldsymbol{y}_{1} & \ldots & \boldsymbol{y}_{N}\end{array}\right]$. In this section, we formulate the problem of finding representative objects from the collection of data points.

\subsection{Learning Compact Dictionaries}

Finding compact dictionaries to represent data has been well-studied in the literature [1, 16, 25, 30, 33]. More specifically, in dictionary learning problems, one tries to simultaneously learn a compact dictionary $\boldsymbol{D}=\left[\begin{array}{lll}\boldsymbol{d}_{1} & \ldots & \boldsymbol{d}_{\ell}\end{array}\right] \in \mathbb{R}^{m \times \ell}$ and coefficients $\boldsymbol{X}=$ $\left[\begin{array}{lll}\boldsymbol{x}_{1} & \ldots & \boldsymbol{x}_{N}\end{array}\right] \in \mathbb{R}^{\ell \times N}$ that can efficiently represent the collection of data points. The best representation of the data is typically obtained by minimizing the objective function

$$
\sum_{i=1}^{N}\left\|\boldsymbol{y}_{i}-\boldsymbol{D} \boldsymbol{x}_{i}\right\|_{2}^{2}=\|\boldsymbol{Y}-\boldsymbol{D} \boldsymbol{X}\|_{F}^{2}
$$

with respect to the dictionary $\boldsymbol{D}$ and the coefficient matrix $\boldsymbol{X}$, subject to appropriate constraints. When the dictionary $\boldsymbol{D}$ is constrained to have orthonormal columns and $\boldsymbol{X}$ is unconstrained, the optimal solution for $\boldsymbol{D}$ is given by the $k$ leading singular vectors of $\boldsymbol{Y}$ [25]. On the other hand, in the sparse dictionary learning framework [1, 16, 30, 33], one requires the coefficient matrix $\boldsymbol{X}$ to be sparse by solving the optimization program

$\min _{\boldsymbol{D}, \boldsymbol{X}}\|\boldsymbol{Y}-\boldsymbol{D} \boldsymbol{X}\|_{F}^{2}$ s.t. $\left\|\boldsymbol{x}_{i}\right\|_{0} \leq s,\left\|\boldsymbol{d}_{j}\right\|_{2} \leq 1, \forall i, j$,

where $\left\|\boldsymbol{x}_{i}\right\|_{0}$ indicates the number of nonzero elements of $\boldsymbol{x}_{i}$ (its convex surrogate can be used as well). In other words, one simultaneously learns a dictionary and coefficients such that each data point $\boldsymbol{y}_{i}$ is written as a linear combination of at most $s$ atoms of the dictionary. Besides being NP-hard due to use of the $\ell_{0}$ norm, this problem is nonconvex because of the product of two unknown and constrained matrices $\boldsymbol{D}$ and $\boldsymbol{X}$. As a result, iterative procedures are employed to find each unknown matrix by fixing the other, which often converges to a local minimizer $[1,16]$.

\subsection{Finding Representative Data}

The learned atoms of the dictionary almost never coincide with the original data [30, 31, 33], hence, can not be considered as good representatives for the collection of data points. To find representative points that coincide with some of the actual data points, we consider a modification to the dictionary learning framework, which first addresses the problem of local minima due to the product of two unknown matrices, i.e., the dictionary and the coefficient matrix. Second, it enforces selecting representatives from the actual data points. To do that, we set the dictionary to be the matrix of data points $\boldsymbol{Y}$ and minimize the expression

$$
\sum_{i=1}^{N}\left\|\boldsymbol{y}_{i}-\boldsymbol{Y} \boldsymbol{c}_{i}\right\|_{2}^{2}=\|\boldsymbol{Y}-\boldsymbol{Y} \boldsymbol{C}\|_{F}^{2}
$$

with respect to the coefficient matrix $\boldsymbol{C} \triangleq\left[\begin{array}{lll}\boldsymbol{c}_{1} & \ldots & \boldsymbol{c}_{N}\end{array}\right] \in$ $\mathbb{R}^{N \times N}$, subject to additional constraints that we describe next. In other words, we minimize the reconstruction error of each data point as a linear combination of all the data. To choose $k \ll N$ representatives, which take part in the linear reconstruction of all the data in (4), we enforce

$$
\|\boldsymbol{C}\|_{0, q} \leq k
$$

where the mixed $\ell_{0} / \ell_{q}$ norm is defined as $\|\boldsymbol{C}\|_{0, q} \triangleq$ $\sum_{i=1}^{N} I\left(\left\|c^{i}\right\|_{q}>0\right)$, where $c^{i}$ denotes the $i$-th row of $C$ and $I(\cdot)$ denotes the indicator function. In other words, $\|\boldsymbol{C}\|_{0, q}$ counts the number of nonzero rows of $\boldsymbol{C}$. The indices of the nonzero rows of $\boldsymbol{C}$ correspond to the indices of the columns of $\boldsymbol{Y}$ which are chosen as the data representatives. Similar to other dimensionality reduction methods, we want the selection of representatives to be invariant with respect to a global translation of the data. We thus enforce the affine constraint $\mathbf{1}^{\top} \boldsymbol{C}=\mathbf{1}^{\top}$. This comes from the fact that if $\boldsymbol{y}_{i}$ is represented as $\boldsymbol{y}_{i}=\boldsymbol{Y} \boldsymbol{c}_{i}$, then for a global translation $\boldsymbol{T} \in \mathbb{R}^{m}$ of the data, we want to have $\boldsymbol{y}_{i}-\boldsymbol{T}=\left[\begin{array}{lll}\boldsymbol{y}_{1}-\boldsymbol{T} & \cdots & \boldsymbol{y}_{N}-\boldsymbol{T}\end{array}\right] \boldsymbol{c}_{i}$.

As a result, to find $k \ll N$ representatives such that each point in the dataset can be represented as an affine combination of a subset of these $k$ representatives, we solve

$$
\min \|\boldsymbol{Y}-\boldsymbol{Y} \boldsymbol{C}\|_{F}^{2} \quad \text { s.t. }\|\boldsymbol{C}\|_{0, q} \leq k, \mathbf{1}^{\top} \boldsymbol{C}=\mathbf{1}^{\top} .
$$

This is an NP-hard problem as it requires searching over every subset of the $k$ columns of $\boldsymbol{Y}$. A standard $\ell_{1}$ relaxation of this optimization is obtained as

$$
\min \|\boldsymbol{Y}-\boldsymbol{Y} \boldsymbol{C}\|_{F}^{2} \text { s.t. }\|\boldsymbol{C}\|_{1, q} \leq \tau, \mathbf{1}^{\top} \boldsymbol{C}=\mathbf{1}^{\top},
$$

where $\|\boldsymbol{C}\|_{1, q} \triangleq \sum_{i=1}^{N}\left\|c^{i}\right\|_{q}$ is the sum of the $\ell_{q}$ norms of the rows of $\boldsymbol{C}$, and $\tau>0$ is an appropriately chosen parameter. ${ }^{1}$ We also choose $q>1$ for which the optimization program in (7) is convex. ${ }^{2}$

The solution of the optimization program (7) not only indicates the representatives as the nonzero rows of $\boldsymbol{C}$, but also provides information about the ranking, i.e., relative importance, of the representatives for describing the dataset. More precisely, a representative that has a higher ranking takes part in the reconstruction of many points in

\footnotetext{
${ }^{1}$ We use $\tau$ instead of $k$ since for the $k$ optimal representatives, $\|\boldsymbol{C}\|_{1, q}$ is not necessarily bounded by $k$.

${ }^{2}$ We do not consider $q=1$ since $\|\cdot\|_{1,1}$ treats the rows and columns equally and does not necessarily favor selecting a few nonzero rows.
} 
the dataset, hence, its corresponding row in the optimal coefficient matrix $\boldsymbol{C}$ has many nonzero elements with large values. On the other hand, a representative with lower ranking takes part in the reconstruction of fewer points in the dataset, hence, its corresponding row in $C$ has a few nonzero elements with smaller values. Thus, we can rank $k$ representatives $\boldsymbol{y}_{i_{1}}, \ldots, \boldsymbol{y}_{i_{k}}$ as $i_{1} \geq i_{2} \geq \cdots \geq i_{k}$, i.e., $\boldsymbol{y}_{i_{1}}$ has the highest rank and $\boldsymbol{y}_{i_{k}}$ has the lowest rank, whenever for the corresponding rows of $\boldsymbol{C}$ we have

$$
\left\|c^{i_{1}}\right\|_{q} \geq\left\|c^{i_{2}}\right\|_{q} \geq \cdots \geq\left\|c^{i_{k}}\right\|_{q} .
$$

Another optimization formulation, which is closely related to (6) is

$$
\min \|\boldsymbol{C}\|_{0, q} \quad \text { s.t. } \quad\|\boldsymbol{Y}-\boldsymbol{Y} \boldsymbol{C}\|_{F} \leq \varepsilon, \mathbf{1}^{\top} \boldsymbol{C}=\mathbf{1}^{\top},
$$

which minimizes the number of representatives that can reconstruct the collection of data points up to an $\varepsilon$ error. An $\ell_{1}$ relaxation of it is given by

$$
\min \|\boldsymbol{C}\|_{1, q} \quad \text { s.t. } \quad\|\boldsymbol{Y}-\boldsymbol{Y} \boldsymbol{C}\|_{F} \leq \varepsilon, \mathbf{1}^{\top} \boldsymbol{C}=\mathbf{1}^{\top} .
$$

This optimization problem can also be viewed in a compression scheme where we want to choose a few representatives that can reconstruct the data up to an $\varepsilon$ error.

\section{Geometry of Representatives}

We now study the geometry of the representative points obtained from the proposed convex optimization programs. We consider the optimization program (10) where we set the error tolerance $\varepsilon$ to zero. First, we show that (10), with a natural additional nonnegativity constraint on $C$, finds the vertices of the convex hull of the dataset. This is, on its own, an interesting result for computing the convex hulls using sparse representation methods and convex optimization. In addition, the robust versions of the optimization program, e.g., $\varepsilon>0$, offer robust approaches for selecting convex hull vertices when the data are perturbed by noise. More precisely, for the optimization program

$$
\min \|\boldsymbol{C}\|_{1, q} \text { s.t. } \boldsymbol{Y}=\boldsymbol{Y} \boldsymbol{C}, \quad \mathbf{1}^{\top} \boldsymbol{C}=\mathbf{1}^{\top}, \quad \boldsymbol{C} \geq \mathbf{0},
$$

we have the following result whose proof is provided in [10].

Theorem 1 Let $\mathcal{H}$ be the convex hull of the columns of $\boldsymbol{Y}$ and let $k$ be the number of vertices of $\mathcal{H}$. The nonzero rows of the solution of the optimization program (11), for $1<$ $q \leq \infty$, correspond to the $k$ vertices of $\mathcal{H}$. More precisely, the optimal solution $C^{*}$ has the following form

$$
\boldsymbol{C}^{*}=\boldsymbol{\Gamma}\left[\begin{array}{cc}
\boldsymbol{I}_{k} & \boldsymbol{\Delta} \\
\mathbf{0} & \mathbf{0}
\end{array}\right],
$$

where $\boldsymbol{I}_{k}$ is the $k$-dimensional identity matrix, the elements of $\boldsymbol{\Delta}$ lie in $[0,1)$, and $\boldsymbol{\Gamma}$ is a permutation matrix.
Theorem 1 implies that, if the coefficient matrix is nonnegative, the representatives are the vertices of the convex hull of the data, $\mathcal{H} .^{3}$ Without the nonnegativity constraint, one would expect to choose a subset of the vertices of $\mathcal{H}$ as the representatives. In addition, when the data lie in a $(k-1)$ dimensional subspace and are enclosed by $k$ data points, i.e., $\mathcal{H}$ has $k$ vertices, then we can find exactly $k$ representatives given by the vertices of $\mathcal{H}$. More precisely, we show the following result [10].

Theorem 2 Let $\mathcal{H}$ be the convex hull of the columns of $\boldsymbol{Y}$ and let $k$ be the number of vertices of $\mathcal{H}$. Consider the optimization program (10) for $1<q \leq \infty$ and $\varepsilon=0$. Then the nonzero rows of a solution correspond to a subset of the vertices of $\mathcal{H}$ that span the affine subspace containing the data. Moreover, if the columns of $\boldsymbol{Y}$ lie in a $(k-1)$-dimensional affine subspace of $\mathbb{R}^{m}$, a solution is of the form

$$
\boldsymbol{C}^{*}=\boldsymbol{\Gamma}\left[\begin{array}{cc}
\boldsymbol{I}_{k} & \boldsymbol{\Delta} \\
\mathbf{0} & \mathbf{0}
\end{array}\right],
$$

where $\Gamma$ is a permutation matrix and the $k$ nonzero rows of $C^{*}$ correspond to the $k$ vertices of $\mathcal{H}$.

\section{Representatives of Subspaces}

We now show that when the data come from a collection of low-rank models, the representatives provide information about the underlying models. More specifically, we assume that the data lie in a union of affine subspaces $\mathcal{S}_{1}, \ldots, \mathcal{S}_{n}$ of $\mathbb{R}^{m}$ and consider the optimization program

$$
\min \|\boldsymbol{C}\|_{1, q} \quad \text { s.t. } \quad \boldsymbol{Y}=\boldsymbol{Y} \boldsymbol{C}, \quad \mathbf{1}^{\top} \boldsymbol{C}=\mathbf{1}^{\top} .
$$

We show that, under appropriate conditions on the subspaces, we obtain representatives from every subspace (left plot of Figure 3) where the number of representatives from each subspace is greater than or equal to its dimension. More precisely, we have the following result [10].

Theorem 3 If the data points are drawn from a union of independent subspaces, i.e., if the subspaces are such that $\operatorname{dim}\left(\oplus_{i} \mathcal{S}_{i}\right)=\sum_{i} \operatorname{dim}\left(\mathcal{S}_{i}\right)$, then the solution of (14) finds at least $\operatorname{dim}\left(\mathcal{S}_{i}\right)$ representatives from each subspace $\mathcal{S}_{i}$. In addition, each data point is perfectly reconstructed by the combination of the representatives from its own subspace.

Since the dimension of the collection of representatives in each subspace $\mathcal{S}_{i}$ is equal to $\operatorname{dim}\left(\mathcal{S}_{i}\right)$, the dimension of the collection of representatives from all subspaces can be as as large as the dimension of the ambient space $m$ by the fact that $\sum_{i} \operatorname{dim}\left(\mathcal{S}_{i}\right)=\operatorname{dim}\left(\oplus_{i} \mathcal{S}_{i}\right) \leq m$.

\footnotetext{
${ }^{3}$ Note that the solution of the $\ell_{1}$ minimization without the affine and nonnegativity constraints is known to choose a few of the vertices of the symmetrized convex hull of the data [8]. Our result is different as we place a general mixed $\ell_{1} / \ell_{q}$ norm on the rows of $\boldsymbol{C}$ and show that for any $q>1$ the solution of (11) finds all vertices of the convex hull of the data.
} 

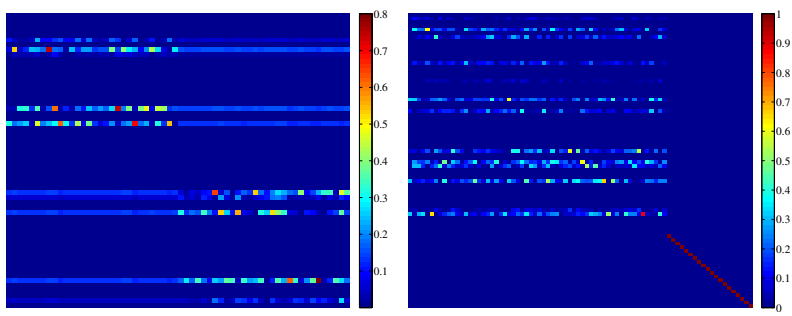

Figure 3. Left: coefficient matrix corresponding to data from two subspaces. Right: coefficient matrix corresponding to a dataset contaminated with outliers. The last set of points corresponds to outliers.

The optimization program (14) can also address the connectivity issues [32] of subspace clustering algorithms based on sparse representation $[11,15,35]$ or low-rank representation [18, 29]. More precisely, as discussed in [15], adding a regularizer of the form $\|\boldsymbol{C}\|_{1,2}$ to the sparse [11] or low-rank [29] objective function improves the connectivity of the points in each subspace, preventing the points in a subspace to be divided into multiple components of the similarity graph.

\section{Practical Considerations and Extensions}

We now discuss some of the practical problems related to finding representative points of real datasets.

\subsection{Dealing with Outliers}

In many real-world problems, the collection of data includes outliers. For example, a dataset of natural scenes, objects, or faces collected from the internet can contain images that do not belong to the target category. A method that robustly finds true representatives for the dataset is of particular importance, as it reduces the redundancy of the data and removes points that do not really belong to the dataset. In this section, we discuss how our method can directly deal with outliers and robustly find representatives for datasets.

We use the fact that outliers are often incoherent with respect to the collection of the true data. Hence, an outlier prefers to write itself as an affine combination of itself, while true data points choose points among themselves as representatives as they are more coherent with each other. In other words, if we denote the inliers by $\boldsymbol{Y}$ and the outliers by $\boldsymbol{Y}_{o} \in \mathbb{R}^{m \times N_{o}}$, for the optimization program

$$
\begin{aligned}
& \min \|\boldsymbol{C}\|_{1, q} \\
& \text { s.t. }\left[\begin{array}{ll}
\boldsymbol{Y} & \boldsymbol{Y}_{o}
\end{array}\right]=\left[\begin{array}{ll}
\boldsymbol{Y} & \boldsymbol{Y}_{o}
\end{array}\right] \boldsymbol{C}, \mathbf{1}^{\top} \boldsymbol{C}=\mathbf{1}^{\top},
\end{aligned}
$$

we expect the solution to have the structure

$$
\boldsymbol{C}^{*}=\left[\begin{array}{cc}
\Delta & \mathbf{0} \\
\mathbf{0} & \boldsymbol{I}_{N_{o}}
\end{array}\right] .
$$

In other words, each outlier is a representative of itself, as shown in the right plot of Figure 3. We can therefore identify the outliers by analyzing the row-sparsity of the solution. Among the rows of the coefficient matrix that correspond to the representatives, the ones that have many nonzero elements correspond to the true data, and the ones that have just one nonzero element correspond to outliers.

In practice, $C^{*}$ might not have exactly the form of (16). However, we still expect that an outlier take part in the representation of only a few other outliers or true data points. Hence, the rows of $C^{*}$ corresponding to outliers should have very few nonzero entries. To detect and reject outliers, we define the row-sparsity index of each candidate representative $\ell$ as

$$
\operatorname{rsi}(\ell)=\frac{N\left\|\boldsymbol{c}^{\ell}\right\|_{\infty}-\left\|\boldsymbol{c}^{\ell}\right\|_{1}}{(N-1)\left\|\boldsymbol{c}^{\ell}\right\|_{1}} \in[0,1] .^{4}
$$

For a row corresponding to an outlier, which has one or a few nonzero elements, the rsi value is close to 1 , while for a row which corresponds to a true representative the rsi is close to zero. Hence, we can reject outliers by selecting representatives whose rsi value is larger than a threshold $\delta$.

\subsection{Dealing with New Observations}

An important problem in finding representatives is to update the set of representative points when new data are added to the dataset. Let $\boldsymbol{Y}$ be the collection of points that has already been in the dataset and $\boldsymbol{Y}_{\text {new }}$ be the new points that are added to the dataset. In order to find the representatives for the whole dataset including the old and the new data, one has to solve the optimization program

$$
\begin{aligned}
& \min \|\boldsymbol{C}\|_{1, q} \\
& \text { s.t. }\left[\begin{array}{ll}
\boldsymbol{Y} & \boldsymbol{Y}_{\text {new }}
\end{array}\right]=\left[\begin{array}{ll}
\boldsymbol{Y} & \boldsymbol{Y}_{\text {new }}
\end{array}\right] \boldsymbol{C}, \mathbf{1}^{\top} \boldsymbol{C}=\mathbf{1}^{\top} .
\end{aligned}
$$

However, note that we have already found the representatives of $\boldsymbol{Y}$, denoted by $\boldsymbol{Y}_{\text {rep }}$, which can efficiently describe the collection of data in $\boldsymbol{Y}$. Thus, it is sufficient to see if the elements of $\boldsymbol{Y}_{\text {rep }}$ are a good representative of the new data $\boldsymbol{Y}_{\text {new }}$, or equivalently, update the representatives so that they can well describe the elements of $\boldsymbol{Y}_{\text {rep }}$ as well as $\boldsymbol{Y}_{\text {new }}$. Thus, we can solve the optimization program

$$
\begin{array}{ll}
\min \|\boldsymbol{C}\|_{1, q} \\
\text { s.t. }\left[\begin{array}{ll}
\boldsymbol{Y}_{\text {rep }} & \boldsymbol{Y}_{\text {new }}
\end{array}\right]=\left[\begin{array}{ll}
\boldsymbol{Y}_{\text {rep }} & \boldsymbol{Y}_{\text {new }}
\end{array}\right] \boldsymbol{C}, \mathbf{1}^{\top} \boldsymbol{C}=\mathbf{1}^{\top},
\end{array}
$$

on the reduced dataset $\left[\begin{array}{ll}\boldsymbol{Y}_{\text {rep }} & \boldsymbol{Y}_{\text {new }}\end{array}\right]$, which is typically of much smaller size than $\left[\begin{array}{ll}\boldsymbol{Y} & \boldsymbol{Y}_{\text {new }}\end{array}\right]$, hence it can be solved more efficiently. ${ }^{5}$

Using similar ideas we can also deal with large datasets using a hierarchical framework. More specifically, we can

\footnotetext{
${ }^{4}$ We use the fact that for $\boldsymbol{c} \in \mathbb{R}^{N}$ we have $\|\boldsymbol{c}\|_{1} / N \leq\|\boldsymbol{c}\|_{\infty} \leq\|\boldsymbol{c}\|_{1}$.

${ }^{5}$ In general, we can minimize $\|\boldsymbol{Q} \boldsymbol{C}\|_{1, q}$, for a diagonal nonnegative matrix $\boldsymbol{Q}$, which gives relative weights to keeping the old representatives and selecting new representatives.
} 


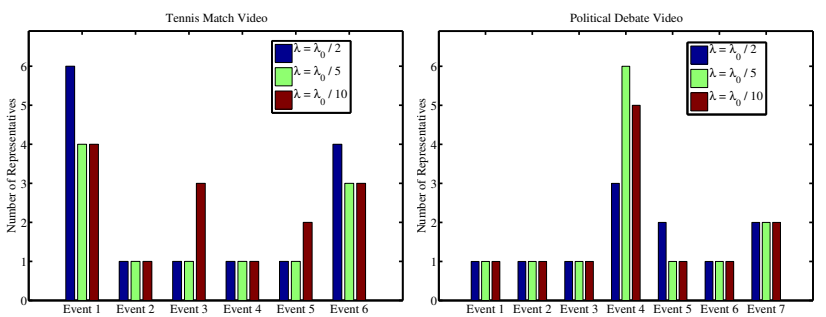

Figure 4. Number of representatives for each event in the video found by our method for several values of the regularization parameter. Left: Tennis match video. Right: Political debate video.

divide $\boldsymbol{Y}$ into $\boldsymbol{Y}_{1}, \ldots, \boldsymbol{Y}_{\ell}$, and find the representatives for each portion of the data, i.e., $\boldsymbol{Y}_{\text {rep }, 1}, \ldots, \boldsymbol{Y}_{\text {rep }, \ell}$. Finally, we can obtain the representatives by solving the proposed optimization programs for $\left[\begin{array}{lll}\boldsymbol{Y}_{\text {rep }, 1} & \ldots & \boldsymbol{Y}_{\text {rep }, \ell}\end{array}\right]$.

\section{Experimental Results}

In this section, we evaluate the performance of the proposed algorithm for finding representatives of real datasets on several illustrative problems. Since, using Lagrange multipliers, either of the proposed optimization programs in (7) or (10) can be written as

$$
\min \lambda\|\boldsymbol{C}\|_{1, q}+\frac{1}{2}\|\boldsymbol{Y}-\boldsymbol{Y} \boldsymbol{C}\|_{F}^{2} \quad \text { s.t. } \mathbf{1}^{\top} \boldsymbol{C}=\mathbf{1}^{\top},
$$

in practice, we use (20) for finding the representatives. We implement the algorithm using an Alternating Direction Method of Multipliers (ADMM) optimization framework [20]. As data points with very small pairwise coherences may lead to too-close representatives, similar to sparse dictionary learning methods [1], one can prune the set of representatives from having too-close data points.

\subsection{Video Summarization}

We first demonstrate the applicability of our proposed algorithm for summarizing videos. First, we consider a 1, 536-frame video taken from [39], which consists of a series of continuous activities with a fixed background (a few frames are shown in Figure 1). We apply our algorithm in (20) and obtain 9 representatives for the whole video. The representatives are shown as frames inside the red rectangles. A summary of the video is provided in the caption of Figure 1. Note that the representatives obtained by our algorithm captured the main events of the video. Perhaps the only missing representative to have a complete description of the whole video is the frame where the man is passing the tiara to the bandit (second row).

Next, we consider a video sequence of a Tennis match (a few frames are shown in Figure 2). The video consists of multiple shots of different scenes where each shot consists of a series of activities. We apply our algorithm in (20) and obtain 11 representatives for the whole video, which are shown in Figure 2 as frames inside the red rectangles. For

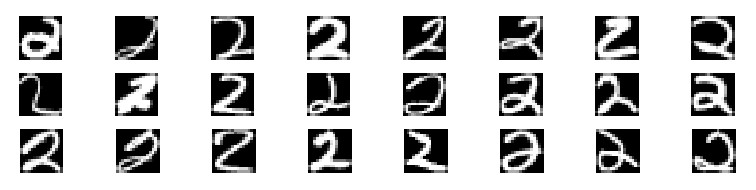

Figure 5. Representatives found by our algorithm for the images of digit 2 . Note that the representatives capture different variations of the digit.

the first and the last shots, which consist of more activities relative to the other shots, we obtain 4 and 3 representative frames, respectively. On the other hand, for the middle shots, which are shorter and have less activities, we obtain a single representative frame.

To investigate the effect of changing the regularization parameter $\lambda$ in the quality of obtaining representatives, we consider the tennis match video as well as a political debate video. We run our proposed algorithm with $\lambda=\lambda_{0} / \alpha$, where $\alpha>1$ and $\lambda_{0}$ is analytically computed from the data [10]. Figure 4 shows the number of representatives found by our method for each of the events in the videos for several values of $\alpha$. Note that first, we always obtain one or several representatives for each of the events. Second, in both videos, the number of representatives for each event does not change much as we change the regularization parameter. Finally, depending on the amount of activities in an event, we obtain an appropriate number of representatives for that event.

\subsection{Classification Using Representatives}

We now evaluate the performance of our method as well as other algorithms for finding representatives that are used for classification. For training data in each class of a dataset, we find the representatives and use them as a reduced training dataset to perform classification. Ideally, if the representatives are informative enough about the original data, the classification performance using the representatives should be close to the performance using all the training data. Therefore, representatives not only summarize a dataset and reduce the data storage requirements, but also can be effectively used for tasks such as classification and clustering.

We compare our proposed algorithm, which we call as Sparse Modeling Representative Selection (SMRS), with several standard methods for finding representatives of datasets: Kmedoids, Rank Revealing QR (RRQR) and simple random selection of training data (Rand). We evaluate the classification performance using several standard classification algorithms: Nearest Neighbor (NN) [9], Nearest Subspace (NS) [22], Sparse Representation-based Classification (SRC) [40], and Linear Support Vector Machine (SVM) [9]. The experiments are run on the USPS digits database [23] and the Extended YaleB face database [28]. ${ }^{6}$ For each class, we randomly select 1,000 (USPS)

\footnotetext{
${ }^{6}$ USPS digits database consists of 10 classes corresponding to handwritten digits $0,1, \ldots, 9$. Extended YaleB face database consists of 38
} 
Table 1. Classification Results on the USPS digit database using 25 representatives of the 1,000 training samples in each class.

\begin{tabular}{|c|c|c|c|c|}
\hline & NN & NS & SRC & SVM \\
\hline \hline Rand & $76.4 \%$ & $84.9 \%$ & $83.5 \%$ & $98.6 \%$ \\
\hline Kmedoids & $\mathbf{8 6 . 0} \%$ & $89.7 \%$ & $89.6 \%$ & $99.2 \%$ \\
\hline RRQR & $59.1 \%$ & $81.3 \%$ & $78.3 \%$ & $94.3 \%$ \\
\hline SMRS & $83.4 \%$ & $\mathbf{9 3 . 8} \%$ & $\mathbf{9 1 . 7} \%$ & $\mathbf{9 9 . 7} \%$ \\
\hline \hline All Data & $96.2 \%$ & $96.4 \%$ & $98.9 \%$ & $99.7 \%$ \\
\hline
\end{tabular}

Table 2. Classification Results on the Extended YaleB face database using 7 representatives of the 51 training samples in each class.

\begin{tabular}{|c|c|c|c|c|}
\hline & NN & NS & SRC & SVM \\
\hline \hline Rand & $30.4 \%$ & $71.3 \%$ & $82.6 \%$ & $87.9 \%$ \\
\hline Kmedoids & $\mathbf{3 7 . 9} \%$ & $80.0 \%$ & $89.1 \%$ & $94.5 \%$ \\
\hline RRQR & $32.2 \%$ & $\mathbf{8 8 . 3} \%$ & $92.9 \%$ & $95.3 \%$ \\
\hline SMRS & $33.8 \%$ & $84.0 \%$ & $\mathbf{9 3 . 1} \%$ & $\mathbf{9 6 . 8} \%$ \\
\hline \hline All Data & $72.6 \%$ & $96.0 \%$ & $98.2 \%$ & $99.4 \%$ \\
\hline
\end{tabular}

/ 51 (YaleB) of the samples for training and obtain the representatives and use the remaining samples in each class for testing. We apply our algorithm in (20) with a fixed $\lambda$ for all classes, which selects, on average, 25 representatives for each class of the USPS database (Figure 5) and 7 representatives for each class of the Extended YaleB database. To have a fair comparison, we select the same number of representatives using Rand, Kmedoids ${ }^{7}$, and RRQR. We also compute the classification performance using all the training data. Tables 1 and 2 show the results for the USPS database and the Extended YaleB database, respectively. From the results, we make the following conclusions:

a- SVM, SRC and NS work well with the representatives found by our method. Note that SRC works well when the data in each class lie in a union of low-dimensional subspaces [12, 14], and, NS works well when the data in each class lie in a low-dimensional subspace. On the other hand, as we discussed earlier, our algorithm can deal with data lying in a union of subspaces, finding representatives from each subspace, justifying its compatibility with both SRC and NS. The good performance of the NS in Table 2 using the representatives obtained by RRQR comes from the fact that the data in each class of the Extended YaleB dataset can be well modeled by a single low-dimensional subspace [3, 28], which is the underlying assumption behind the RRQR algorithm for finding the representatives.

b- For the NN method to work well, we often need to have enough samples from each class so that given a test sample, its nearest neighbor comes from the right class. Thus, methods such as Kmedoids that look for the centers of the data distribution in each class perform better with NN. For

classes of face images corresponding to different individuals captured under a fixed pose and varying illumination.

${ }^{7}$ Since Kmedoids depends on initialization, we use 100 restarts of the algorithm and take the result that obtains the minimum energy.
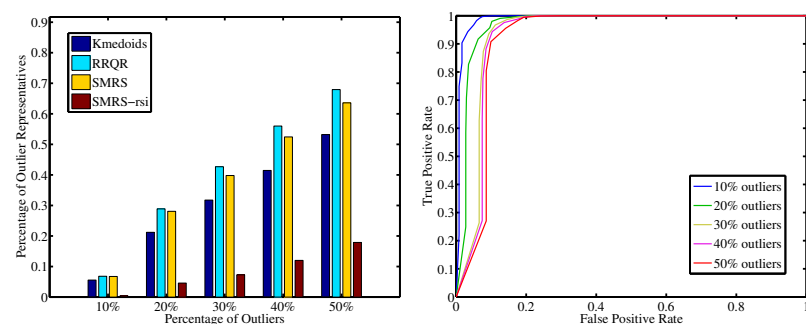

Figure 6. Left: percentage of outlier representatives for different algorithms as a function of the percentage of outliers in the dataset. Right: ROC curves for the proposed method for different percentages of outliers.

the NN method in the Extended YaleB dataset, the large gap between using all the training data and using the representatives obtained by different algorithms is mainly due the fact that the data in different classes are close to each other $[13,15]$, hence using a subset of the the training data can significantly change the inter and intra class distances of the training data.

\subsection{Outlier Rejection}

To evaluate the performance of our algorithm for rejecting outliers, we form a dataset of $N=1,024$ images, where $(1-\rho)$ fraction of the images are randomly selected from the Extended YaleB face database. The remaining $\rho$ fraction of the data, which correspond to outliers, are random images downloaded from the internet. For different values of $\rho$ in $\{0.1,0.2,0.3,0.4,0.5\}$, we run our algorithm as well as Kmedoids and RRQR to select roughly 100 representatives for the dataset. Figure 6 (left) shows the percentage of outliers among the representatives as we increase the number of outliers in the dataset. We show the result of our algorithm prior to and after rejecting representatives using rsi $>\delta$, where for all values of $\rho$ we set $\delta=0.16$. As expected, the percentage of outliers among representatives increases as the number of outliers in the dataset increases. Also, all methods, select roughly the same number of outliers in their representatives. However, note that our proposed algorithm has the advantage of detecting and rejecting outliers by simply analyzing the row-sparsity of the coefficient matrix $\boldsymbol{C}$. As shown in the plot, by removing the representatives whose rsi value is greater than $\delta=0.16$, the number of outlier representatives significantly drops for our algorithm (we still keep at least $90 \%$ of the true representatives as shown in the ROC curves). Figure 6 also shows the ROC curves of our method for different percentages of outliers in the dataset. Note that, for all values of $\rho$, we can always obtain a high positive rate, i.e., keep many true representatives, with a relatively low false positive rate, i.e., select very few outliers in the representatives.

\section{Discussion}

We proposed an algorithm for finding a subset of the data points in a dataset as the representatives. We assumed that 
each data point can be expressed efficiently as a combination of the representatives. We cast the problem as a joint sparse multiple measurement vector problem where both the dictionary and the measurements are given by the data points and the unknown sparse codes select the representatives. For a convex relaxation of the original nonconvex formulation, we showed the relationship of the representatives to the vertices of the convex hull of the data. It is important to note that the convex relaxation takes into account the value of the norm of the coefficients, hence prefers representatives with such geometrical properties. As we show in [10], greedy algorithms that are insensitive to the norm of the coefficients lead to representatives with different geometrical properties. When the data come from a collection of low-rank models, under appropriate conditions, we showed that our proposed algorithm selects representatives from each low-rank model. It is important to note that our proposed algorithm also allows to incorporate the prior knowledge about the nonlinear structure of the data using kernel methods and weighting the coefficient matrix into the optimization program [10].

\section{Acknowledgment}

E. Elhamifar would like to thank Ewout van den Berg for fruitful discussions about the paper. E. Elhamifar and R. Vidal are supported by grants NSF CNS-0931805, NSF ECCS-0941463, NSF OIA-0941362, and ONR N00014-09-10839. G. Sapiro acknowledges the support by DARPA, NSF, and ONR grants.

\section{References}

[1] M. Aharon, M. Elad, and A. M. Bruckstein. The k-svd: An algorithm for designing of overcomplete dictionaries for sparse representations. IEEE TIP, 2006. 2, 3, 6

[2] L. Balzano, R. Nowak, and W. Bajwa. Column subset selection with missing data. NIPS Workshop on Low-Rank Methods for Large-Scale Machine Learning, 2010. 1

[3] R. Basri and D. Jacobs. Lambertian reflection and linear subspaces. IEEE TPAMI, 2003. 7

[4] S. Bengio, F. Pereira, Y. Singer, and D. Strelow. Group sparse coding. NIPS, 2009. 2

[5] C. Boutsidis, M. W. Mahoney, and P. Drineas. An improved approximation algorithm for the column subset selection problem. Proceedings of SODA, 2009. 1

[6] T. Chan. Rank revealing qr factorizations. Lin. Alg. and its Appl., 1987. 1

[7] R. Coifman and S. Lafon. Diffusion maps. Applied and Computational Harmonic Analysis, 2006. 1

[8] D. L. Donoho. Neighborly polytopes and sparse solution of underdetermined linear equations. (preprint), 2004. 4

[9] R. Duda, P. Hart, and D. Stork. Pattern Classification. WileyInterscience, 2004. 6

[10] E. Elhamifar, G. Sapiro, and R. Vidal. Sparse modeling for finding representative objects. in preparation. $4,6,8$

[11] E. Elhamifar and R. Vidal. Sparse subspace clustering. CVPR, 2009. 2, 5

[12] E. Elhamifar and R. Vidal. Robust classification using structured sparse representation. CVPR, 2011. 7
[13] E. Elhamifar and R. Vidal. Sparse manifold clustering and embedding. NIPS, 2011. 7

[14] E. Elhamifar and R. Vidal. Block-sparse recovery via convex optimization. IEEE TSP, 2012. 7

[15] E. Elhamifar and R. Vidal. Sparse subspace clustering: Algorithm, theory, and applications. IEEE TPAMI, submitted., Available: http://arxiv.org/abs/1203.1005. 2, 5, 7

[16] K. Engan, S. O. Aase, and J. H. Husoy. Method of optimal directions for frame design. ICASSP, 1999. 3

[17] E. Esser, M. Moller, S. Osher, G. Sapiro, and J. Xin. A convex model for non-negative matrix factorization and dimensionality reduction on physical space. Technical report, Available: http://arxiv.org/abs/1102.0844, 2011. 1, 2

[18] P. Favaro, R. Vidal, and A. Ravichandran. A closed form solution to robust subspace estimation and clustering. CVPR, 2011. 2, 5

[19] B. J. Frey and D. Dueck. Clustering by passing messages between data points. Science, 2007. 1

[20] D. Gabay and B. Mercier. A dual algorithm for the solution of nonlinear variational problems via finite-element approximations. Comp. Math. Appl., 1976. 6

[21] M. Gu and S. C. Eisenstat. Efficient algorithms for computing a strong rank-revealing qr factorization. SIAM Journal on Scientific Computing, 1996. 1

[22] J. Ho, M. H. Yang, J. Lim, K. Lee, and D. Kriegman. Clustering appearances of objects under varying illumination conditions. CVPR 2003. 6

[23] J. J. Hull. A database for handwritten text recognition research. IEEE TPAMI, 1994. 6

[24] R. Jenatton, J. Y. Audibert, and F. Bach. Structured variable selection with sparsity-inducing norms. JMLR, 2011. 2

[25] I. Jolliffe. Principal Component Analysis. Springer, 2002. 1, 3

[26] L. Kaufman and P. Rousseeuw. Clustering by means of medoids. In Y. Dodge (Ed.), Statistical Data Analysis based on the L1 Norm (North-Holland, Amsterdam), 1987. 1

[27] N. Keshava and J. Mustard. Spectral unmixing. IEEE Signal Processing Magazine, 2002. 1

[28] K. C. Lee, J. Ho, and D. Kriegman. Acquiring linear subspaces for face recognition under variable lighting. IEEE TPAMI, 2005. 6, 7

[29] G. Liu, Z. Lin, and Y. Yu. Robust subspace segmentation by low-rank representation. ICML, 2010. 2, 5

[30] J. Mairal, F. Bach, J. Ponce, G. Sapiro, and A. Zisserman. Discriminative learned dictionaries for local image analysis. CVPR, 2008. 2, 3

[31] J. Mairal, F. Bach, J. Ponce, G. Sapiro, and A. Zisserman. Non-local sparse models for image restoration. ICCV, 2009. 2, 3

[32] B. Nasihatkon and R. Hartley. Graph connectivity in sparse subspace clustering. In $C V P R, 2011.5$

[33] I. Ramirez, P. Sprechmann, and G. Sapiro. Classification and clustering via dictionary learning with structured incoherence and shared features. CVPR, 2010. 2, 3

[34] S. Roweis and L. Saul. Nonlinear dimensionality reduction by locally linear embedding. Science, 2000. 1

[35] M. Soltanolkotabi and E. J. Candes. A geometric analysis of subspace clustering with outliers. Available: http://arxiv.org/abs/1112.4258. 5

[36] J. B. Tenenbaum, V. de Silva, and J. C. Langford. A global geometric framework for nonlinear dimensionality reduction. Science, 2000. 1

[37] J. A. Tropp. Algorithms for simultaneous sparse approximation. part ii: Convex relaxation. Signal Processing, special issue "Sparse approximations in signal and image processing", 2006. 2

[38] J. A. Tropp. Column subset selection, matrix factorization, and eigenvalue optimization. Proceedings of SODA, 2009. 1

[39] R. Vidal. Recursive identification of switched ARX systems. Automatica, 2008. 6

[40] J. Wright, A. Yang, A. Ganesh, S. Sastry, and Y. Ma. Robust face recognition via sparse representation. IEEE TPAMI, 2009. 6 